\title{
CAMBIOS SOCIALES Y EVALUACIÓN DE LA EDUCACIÓN EN MÉXICO. EL CASO DE LA LECTURA
}

Mutra. Silvia Nicolás Cisnerost, Mtra. Alma Ludia Martinez: Castro**, Dra. Coralia Pérez Maya***

* Profesora de Carrera Asociado "C", ENEO-UNAM, "* Profesora de Tiempo Completo en el Centro Cuitural Universitario Justo Sierra y Universidad Mesoamericana, *** Profesora investigadora del Area de Ciencias de la Educación del Instituto de Ciencias Saciales y Humanidades de la Universidad Autónama del Estado de Hidaigo.

\section{RESUMEN:}

A partir de la lectura de documentos oficiales, se reflexiona sobre los cambios que ha presentado el Estado mexicano, para asumirse actualmente como un estado evaluador; la participación de la Organización de Cooperación para el Desarrollo Económico' (OCDE), en el diagnóstico sobre la educación en México, y la aplicación del Programa Internacional de Evaluación de Estudiantes (PISA), sus resultados y la respuesta que da el gobierno en la generación de estrategias para cambiar aptitudes de lectura de la población mexicana.

Se presentan resultados de una encuesta aplicada en el 2005, por la Procuraduría Federal del Consumidor (Profeco), para identificar cómo, a ocho años del diagnóstico de la OCDE (1997) y a cinco de la aplicación del PISA (2000), con énfasis en la lectura, la población sigue presentando resultados negativos.

La propuesta es ya no esperar políticas públicas, ante esta necesidad urgente, adoptar una actitud reflexiva y responsable con nosotros mismos y la población con la que nos relacionamos, ya sea por razones laborales o familiares; con la certidumbre de que somos capaces de construir mejores mañanas.

Palabras clave: cambios sociales, evaluación educativa, habilidad lectora.

\section{SUMMARY}

Starting from the reading of official documents, one can reflect about the changes the Mexican Government has presented for assuming itself as an evaluating state; about the Cooperative Organization for the Economical Development (OCDE) participation to the diagnostics about education in Mexico, and the Students Evaluation International Program (PISA) application, their outcomes, and the answer given by the government when generating strategies to change the Mexican population reading abilities.

Here, the outcomes of a survey carried out in 2005 by the Federal Procurator Office for Consumers (Profeco) in order to identify how, at eight years from the OCDE diagnoses (1997) and at five from the PISA application (2000), with an emphasis on reading, population continues showing negative outcomes.

The proposal is to stop waiting for public politics before this urgent need and realize a reflexive and responsible attitude must be adopted with ourselves and with the population with which we are relating, either because working or family reasons; with the certitude that we are capable to build a better "tomorrow".

Key words: social changes, evaluation of education, reading abilities. 


\section{INTRODUCCIÓN}

Ya desde 1959, Peter F. Drucker, en su obra Landmarks of tomorow señaló no solo a la educación, sino a la "alta educación" como "requisito previo absoluto del desarrollo social y económico en nuestro mundo". Resalta Drucker, que para tener una sociedad o una economía desarrolladas no basta con que las personas pasen largos años en la escuela, que ya no es posible seguir educando por debajo del limite de las capacidades de las personas.

En los 80s, México había logrado incrementar la cobertura de la educación superior, con programas innovadores, muestra de esto los tenemos en las Escuelas Nacionales de Estudios Profesionales de la Universidad Nacional Autónoma de México (UNAM) y los planteles de la Universidad Autónoma Metropolitana (UAM), en este tiempo se consolida un crecimiento de las instituciones de Educación Superior (IES), apoyado por políticas gubernamentales. Vino entonces la crisis del 82, que afectó al sistema educativo; que se ve cada vez más cuestionado; se debilita al sector estatal como generador de empleo, y como resultado se genera una política que busca la articulación del sector educativo con el sector laboral privado, posicionándose el Estado como regulador de los mercados laborales, mediante la acreditación de las IES, por medio de la revisión de los planes y programas educativos asi como de las características de sus recursos y organización institucional; además de la certificación profesional que avala su ejercicio.

Esta nueva relación de las IES, el Estado y la sociedad mexicana, convierten al segundo en un Estado evaluador de la denominada calidad de los procesos del mercado educativo o sea, de la educación universitaria, y de sus productos, es decir, sus egresados.

En lo internacional, encontramos una forma diferente pero complementaria, de evaluación de la educación en México. Para este análisis se presenta el caso de la OCDE. En el 2002, Pérez Maya señala respecto a esta participaciôn: "Algunos organismos internacionales han invertido grandes esfuerzos en la definición de sistemas de indicadores internacionales de la educación, con el convencimiento de contribuir asi a mejorar el conocimiento que tienen políticos y autoridades de la educación de sus propios sistemas con respecto a otros que pueden tomar por comparables y, por consiguiente como referentes."

El año de 1997, la OCDE; presentó el texto Exámenes de las políticas nacionales de educación. México, Educación Superior. En la primera parte encontramos el informe.
Básico, que realizaron autoridades mexicanas a la OCDE en la segunda parte presentan el Informe de los examina. dores de la OCDE y al final las Recomendaciones. Es un texto sumamente interesante en las dos primeras partes a ocho años de su publicación, encontramos un diagnóstico aún vigente; y la lectura de la tercera parte permite orientar el análisis sobre la forma en que se ha venido aplicando la política educativa en nuestro país. Su com prensión también permite captar la importancia de cono. cer los resultados de las evaluaciones internacionales que aplica la misma OCDE.

En la revisión de los documentos 1997 y 2000 de la OCDE, se encuentra una clara relación del diagnóstico con los indicadores que plantea, aplica y evalúa el PISA?. En PISA 2000, se midieron -Aptitudes para la lectura, las matemáticas y las ciencias-. En la primera aplicación (PISA 2000) se privilegió el área de lectura, dicha evaluación fue aplicada en 25 paises; ampliando la recogida de datos sobre las aptitudes hacia el aprendizaje e información y cómo manejan los estudiantes su propio aprendizaje. PISA 2003. tuvo un enfoque principal hacia las matemáticas. PISA 2006, tendrá un enfoque principal hacia las ciencias y PISA 2009, nuevamente hacia la lectura.

Los resultados respecto a la lectura, que se encuentran en el informe de PISA 2000, destacan lo siguiente: en tres paises de la OCDE, entre ellos México, más del $25 \%$ de la población del estudio, (jóvenes de 15 años) se sitúan en el Nivel 1 o por debajo, es decir desarrollaron únicamente las tareas más sencillas que se asocian con la habilidad lectoras

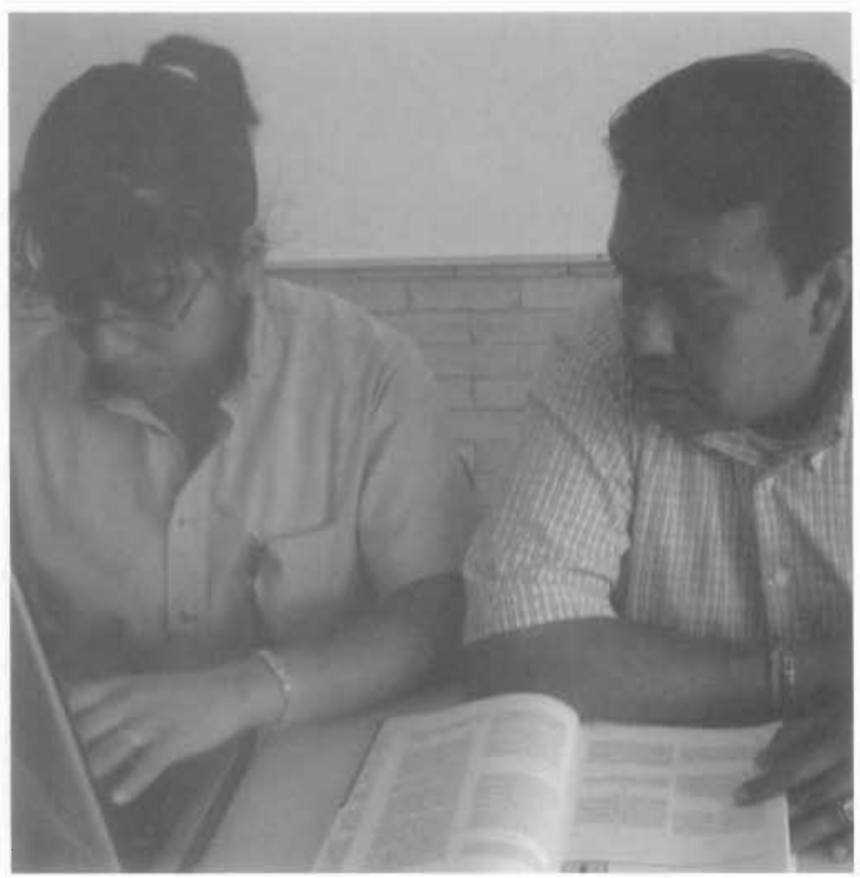


Es claro que el gobierno actual, ha tomado en consideración los documentos anteriores para generar sus políticas y sus programas. Ya deșde el documento presentado por el equipo de transición a la entrada del actual gobierno, Bases para el programa sectorial de educación 20012006, en la parte correspondiente al Diagnóstico de los problemas, se encuentra una gran coincidencia de: datos, con el Informe presentado por los examinadores de la OCDE en 1997.

El Plan Nacional de Desarrollo 2001-2006, en el plano discursivo, presenta a la educación como el gran proyecto nacional, para elevar la competitividad del país en el entorno global, ampliar la capacidad de los ciudadanos y aumentar los niveles de calidad de vida de la población. Es decir el combate a la pobreza, mediante la educación, sin embargo no se encuentran en el documento oficial las políticas, estrategias o acciones del sector, que le darían viabilidad al proyecto.

\section{REVISTA DEL CONSUMIDOR}

Lo anterior ha sido un pequeño recorrido para reconocer que a 5 años de aplicada la primera evaluación PISA 2000, Programa Internacional de Evaluación de Estudiantes, con enfoque en las aptitudes para la lectura, y a cuatro de que se realice la próxima con este mismo enfoque en 2009; las condiciones de la política y sus estrategias, las que se hayan generado, han dado pobres resultados, que confirman lo dicho en 2001, por Prawda y Flores: "Por sí solos, los sistemas de medición no mejo-

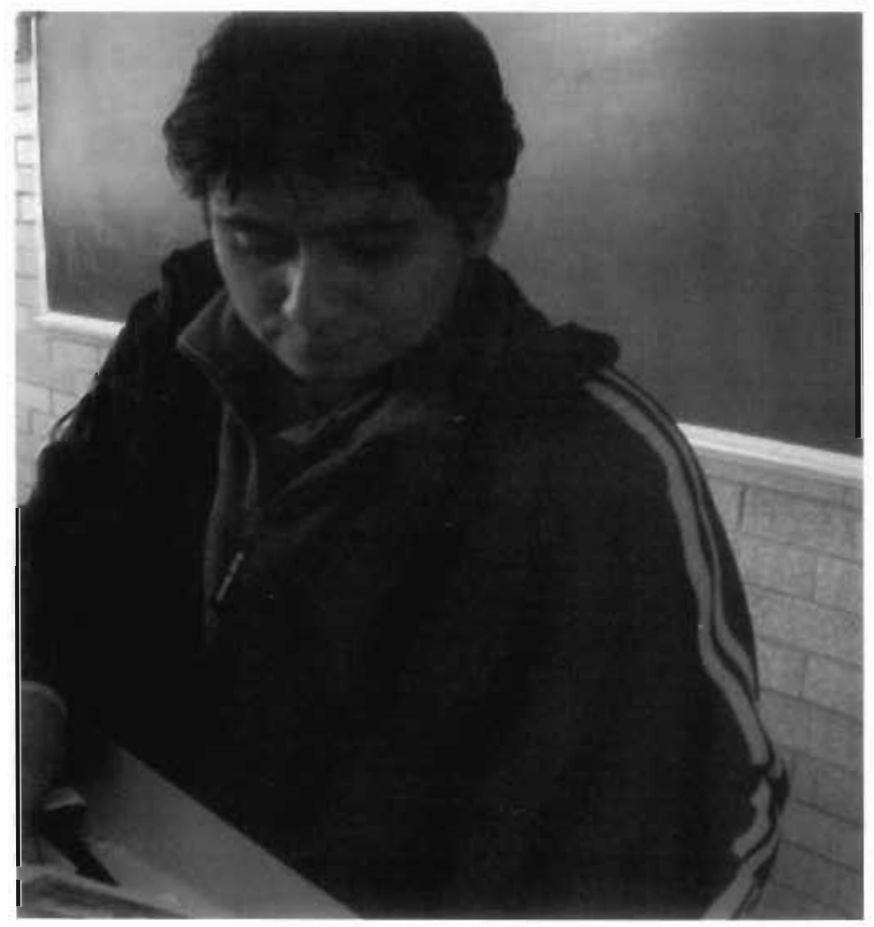

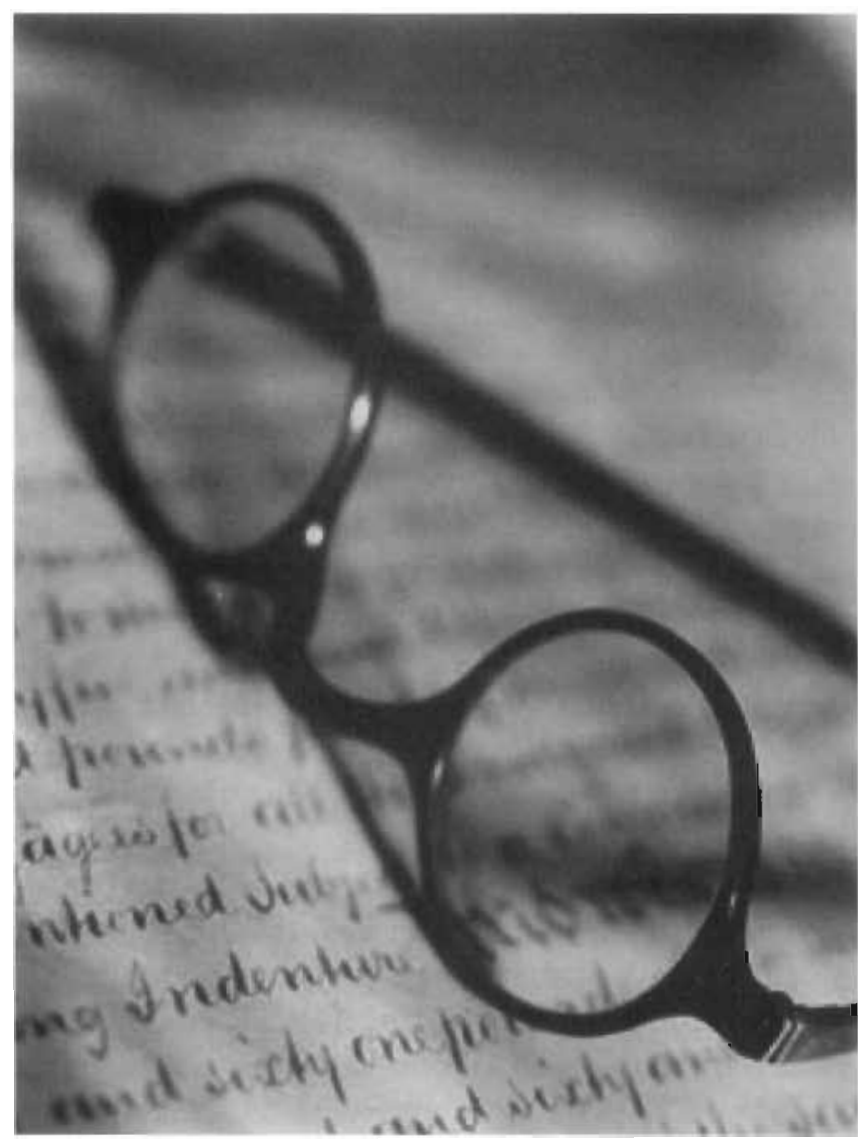

ran la efectividad de un sistema educativo. Son condiciones necesariass pero no suficientes para hacerlo"

Los resultados que se presentan a continuación fueron obtenidos del artículo de Julieta Arévaio, en la Revista del Consumidor de Julio, 2005. Por la importancia del tema y el impacto en el trabajo de los profesionales de la educación y de la salud, que trabajamos con esta población, consideramos de interés conocerios, analizarlos y en nuestra comunidad, familiar, laboral; en el ámbito de nuestra competencia, darles alguna salida.

La Dirección General de Estudios sobre Consumo de PROFECO, realizó una entrevista, en abril de 1995, a 1,133 mexicanos (as) mayores de 17 años, residentes en el área metropolitana de la Ciudad de México, para saber cuánto leen, qué leen y porqué. Los resultados podemos considerarlos representativos de las diferentes comunidades, como nuestros alumnos, ruestros familiares o las comunidades a las que atendemos, o tal vez como nosotros mismos.

Presentamos a continuación, una selección de los datos, considerados los más interesantes, con la invitación a leer completo el artículo, en impreso o en la página web de la Profeco. 


\begin{tabular}{ccccccc}
\multicolumn{1}{c}{ CUADRO 1 } \\
COMPARATIVO INTERNACIONAL DE PERSONAS QUE TIENEN EL HÁBITO DE LECTURA \\
JAPÓN & ALEMANIA & FRANCIA & ESPAÑA & CHILE & ARGENTINA & MÉXICO \\
$91 \%$ & $60 \%$ & $57 \%$ & $39 \%$ & $18 \%$ & $17 \%$ & $2 \%$
\end{tabular}

Fuente: Eduardo Robes Boza, Si no leo me a-burro. Grijalbo, México, 2000. Tomado de la Revista del Consumidor.

\section{CUADRO 2}

COMPARATIVO INTERNACIONAL DE LIBRERIAS POR HABITANTES

MÉXICO

Una por cada 200

mil habitantes

(MILES)

$\begin{array}{ll}\text { ARGENTINA } & \text { FRANCIA } \\ \text { Una por cada } 14 & \text { Una por cada } 10 \\ \text { mil habitantes } & \text { mil habitantes }\end{array}$

ESPAÑA

Una por cada 8

mil habitantes

Fuente: Adriana Malvado, "Los libros: mundo de imaginación en peligro", en La Jornada 13 de diciembre 1993. Tomado de la Revista del Consumidor.

\section{CUADRO 3 \\ AUTORES MÁS LEÍDOS, POR ORDEN DE FRECUENCIA}
1. Gabriel Carcía Márquez
2. Carlos Cuauhtémoc Sánchez
3. Amado Nervo
4. Carlos Fuentes
5. Octavio Paz
6. Pablo Neruda
7. Edgar Allan Poe
8. Paulo Coelho
10. Juan Rulfo
11. Mario Benedetti
9. Isabel Allende
13. William Shakespeare
14. Jorge Luis Borges
12. Dan Brown
15. José Saramago

Fuente: Dirección General de Estudios sobre Consumo, Profeco. Encuesta realizada del 13 al 21 de abril de 2005 entre 1,133 personas mayores de 17 años que viven en el área metropolitana de la Ciudad de México. La encuesta se aplicó a población abierta. Tiene un nivel de confianza del $95 \%$ y un margen de error de $+\ldots .7 \%$.

\section{CUADRO 4}

TIPOS DE LIBROS QUE LAS PERSONAS LEEN POR INICIATIVA PROPIA

$\begin{array}{lllllllll}\text { Literatura } & \text { Ciencias } & \text { Superación } & \text { Ciencia y } & \text { Libros } & \text { De } & \text { Libros } & \text { Libros } & \text { Otro } \\ \text { general } & \text { sociales } & \text { personal } & \text { tecnología } & \text { prácticós } & \text { todo } & \text { religiosos } & \text { de arte } & \\ 41 \% & 18.6 \% & 16.7 \% & 9.3 \% & 4.5 \% & 4.1 \% & 2.7 \% & 2.4 \% & 0.7 \%\end{array}$

Fuente: Dirección General de Estudios sobre Consumo, Profeco. Encuesta realizada del 13 al 21 de abril de 2005 entre 1,133 personas mayores de 17 años que viven en el área metropolitana de la Ciudad de México. La encuesta se aplicó a población abierta. Tiene un nivel de confianza del $95 \%$ y un margen de error de +_ $1.8 \%$.

$\begin{array}{lc}\text { CUADRO 5 } \\ \text { GÉNERO LITERARIO QUE LAS PERSONAS LEEN POR INICIATIVA PROPIA } \\ \text { GÉNERO LITERARIO } & \% \\ \text { NARRATIVA } & 32 \\ \text { HISTÓRICA } & 27 \\ \text { MITOS Y LEYENDAS } & 12 \\ \text { TERROR Y SUSPENSO } & 9 \\ \text { CIENCIA FICCIÓN } & 9 \\ \text { NO SABE QUE ES UN GÉNERO LITERARIO } & 5 \\ \text { NO CONTESTÓ } & 5 \\ \text { FANTÁSTICA } & 3 \\ \text { POLICIACA } & 2 \\ \text { OTRO } & 2\end{array}$

Fuente: Dirección General de Estudios sobre Consumo, Profeco. Encuesta realizada del 13 al 21 de abril de 2005 entre 1,133 personas mayores de 17 años que viven en el área metropolitana de la Ciudad de México. La encuesta se aplicó a población abierta. Tiene un nivel de confianza del 95\% y un margen de error de ${ }_{-} 1.8 \%$. 


\section{Los Maestros Escriben}

Para el análisis, identificamos casos extremos, en un polo se encuentran los casi seis millones de analfabetos reportados por el INEGI el año 2000, además están los analfabetos funcionales, quienes no leen o no captan los mensajes escritos, de ahí el éxito de los "monitos o comics", con mínimos contenidos escritos, que si bien estimulan la imaginación, no crean una cultura lectora.

En el otro extremo, resaltan a un encuestado, estudiante de Filosofía en la UNAM, quién lee por tarea y lee por placer, cuando menos media hora en sus ratos libres; aún si reconocemos que el tiempo que le asigna a la lectura es poco, es superior a la media estudiada, de este dato surgen algunas preguntas; ¿Qué estilo de pensamiento se construye en esta disciplina?, ¿Qué otros factores han incidido en las motivaciones para la lectura que presenta este joven?

Es preocupante que los encuestados, manifiesten rechazo por la lectura (el 63\% de los entrevistados reconocen que su gusto por la lectura va de regular a nulo); que señalen de primera instancia que no leen por la saturación de tareas escolares ( $40 \%$ de los encuestados señaló que no leen por no tener tiempo), sin embargo, para cuando reflexionan, expresan que pese a conocer los beneficios de la lectura (60\% de los encuestados afirmó que le gustaría leer más), sus aficiones son la televisión o los videojuegos. La reportera Arévalo señala "Tristemente la educación formal incentiva muy poco la lectura de textos adicionales a los que forman parte de los programas oficiales de estudio".

Lo anterior tiene que ver con lo que también los encuestados señalan, en la educación formal, hay una evaluación del momento, solo lo objetivo, sin discusión del reporte de lectura, sin identificación de los sentidos del texto, sin un seguimiento de la evolución de las capacidades, "Esto sucede cada bimestre, cuando leemos por nuestra calificación".

Dentro de las notas resaltan que de acuerdo a la Cámara Nacional de la industria Editorial, el promedio de la población mexicana lee 1.8 libros anuales. Es muy poco si queremos participar en la "era del conocimiento", algunos lo podrán hacer, tendrán las competencias y capacidades operativas. Para nuestra sociedad ¿Qué vamos a construir?

Existe una coincidencia sobre la corresponsabilidad de la familia y la escuela, a las instituciones también les toca esforzarse más, hay que reconocer que se han hecho esfuerzos, programas de bibliotecas públicas, publicaciones a bajo costo, diversificación de temas por las editoria- les, en la ENEO, existe la Biblioteca "Graciela Arroyo de Cordero" actualizada, cada día más completa, iriquisima!.

\section{CONCLUSIONES}

Desde el cambio social, México se inscribe dentro del modelo de Estado evaluador, sin embargo, respecto al tema del presente ensayo - la lectura-, no se han tomado las decisiones que permitan corregir las insuficiencias detectadas. Nos encontramos dentro de la sociedad global, en donde hay posibilidades por parte de los gobiernos de los diferentes países a aplicar por voluntad, políticas diferentes a las recomendadas por los organismos internacionales. Hay un reconocimiento que los diagnósticos, las miradas, las propuestas, los modelos de la investigación comparada aplicada por organismos internacionales, son valiosos, por el esfuerzo realizado, dignas de aprovechar sin ser tomadas como recetas mágicas o leyes inamovibles, sin embargo, respecto a la lectura, en nuestro país, no se han tomado las decisiones que permitan corregir las insuficiencias detectadas, hay la necesidad de oponer al pensamiento mecanicista la cultura de la participación, con argumentos sólidos, resultado de investigaciones, con flexibilidad y racionalidad reflexiva y crítica. Desde la colegialidad y comprendiendo la resistencia, los diversos actores han de construir diferentes escenarios, para que los esfuerzos continuados y sistemáticos den mejores mañanas.

Retomamos a Drucker, para señalar que aún más que en su época (1959), en la sociedad del conocimiento actual (2005), la revolución educativa consiste en la transformación de lo que antaño se consideró un ocio - el saber-; hoy el trabajo de saber y el capital en que se convierte, ya no es improductivo en cuanto a bienes y servicios, el capital del saber es el capital verdadero de la sociedad a en la que deseamos participar, y una vía regia de entrada al saber es la lectura.

Sobre la cultura lectora, es imprescindible quitarse la idea que quien lee pierde el tiempo, comprender y guiar en la realización de esta tarea intelectual; facilitar las condiciones para el trabajo con ideas, que le permitirán indagar permanentemente sobre el mundo y sus interacciones, para poder anticipar y construir realidades profesionales y cotidianas, quien lee está llevando un diálogo con el autor y conociendo otra forma de ver el mundo, que lo enriquecerá para siempre.

Con el enfoque del constructivismo de Peter Berger y Thomas Luckmann (1968), señalamos que toda sociedad humana es una empresa de construcción del 
mundo, que la sociedad como fenómeno dialéctico, es producto de la persona, quién a la vez es producto de la sociedad. Que la cultura es producida y reproducida por el hombre (y las mujeres), y que la actividad humana de construcción del mundo es siempre una empresa colectiva. Con el constructivismo podemos coincidir "que un mundo mejor es posible".

\section{NOTAS DE REFERENCIA}

1 Organismo que agrupa a las naciones desarrolladas; a las que, por cuestiones politicas pertenece nuestro pais a partir de 1994

2 Mucho antes de que México participara, el Consejo de la OCDE creó en 1968 el Centro para la Investigación e innovación en la Enseñanza, con los siguientes objetivos:

"-favorecer y apoyar el desarrollo de las actividades de investigación relacionadas con la educación y emprender, llegado el caso, actividades de esta naturaleza.

-favorecer y apoyar experiencias piloto con vistas a introducir y probar innovaciones en la enseñanza.

-favorecer el desarrollo de la cooperación entre los países miembros en el campo de la investigación y la innovación en la enseñanza" (OCDE. 1996:2)

3 Las tareas lectoras desarrolladas por el Proyecto PISA son: recuperar información, interpretar la leido, reflexionar sobre el texto y valorar el texto; basándose en sus conocimientos previos.

\section{REFERENCIAS BIBLIOGRÁFICAS}

Berger - L; P y Luckmann; T. La construcción social de la realidad. Arnorrortu. Argentina, 1968. 233 pp.
Drucker; P. "La revolución educativa". En: Etzioni; A. y Etzioni; E. Los cambios sociales. Fondo de Cultura Económica, México, 1968. 453 pp.

Loría - M. Eduardo. La competitividad de las universidades públicas mexicanas. Una propuesta de evaluación. UAEM/Plaza y Valdés, México, 2002. 153 pp.

Mendoza - R. Javier. Transición de la educación superior contemporánea en México: de la planeación al Estado evaluador. CESU UnAM/Miguel Ángel Porrúa, México, 2002. 358 pp.

OCDE Panorama educativo. Análisis 1996. Indicadores de los Sistemas de enseñonza. Centro para la investigación e Innovación en la Enseñanza. Francia. 1996. 79 pp

OCDE Exámenes de las politicas nacionales de educación. Méxica. Educación Superior. Francia, 1997. 242 pp.

OCDE informe sobre el desempeño de los estudiontes. Programa Internacional de Evaluación de Estudiantes. PISA 2000. Francia, 2000. 237 pp.

Pérez - M. Coralia y Cols "ccuál es el papel de la evoluación educativa?". Ponencia en: $2^{\circ}$ Coloquio Regional de Investigoción en Posgrados en Ciencias Sociales y Humanidades. Hidalgo, Tlaxcala y Puebla. México, 2002, 10 pp.

Prawda; Juan y Flores; G. México educativo revisitado. Océano, México, 2001317 pp.

\section{DIRECCIÓN PARA CORRESPONDENCIA}

Silvia Nicolás Cisneros: ssnc@servidor.unam.mx.

Alma Lucia Martinez Castro: email mafufa2000@hotmail.com

Coralia Pérez Maya: cpm258@yahoo.com.mx

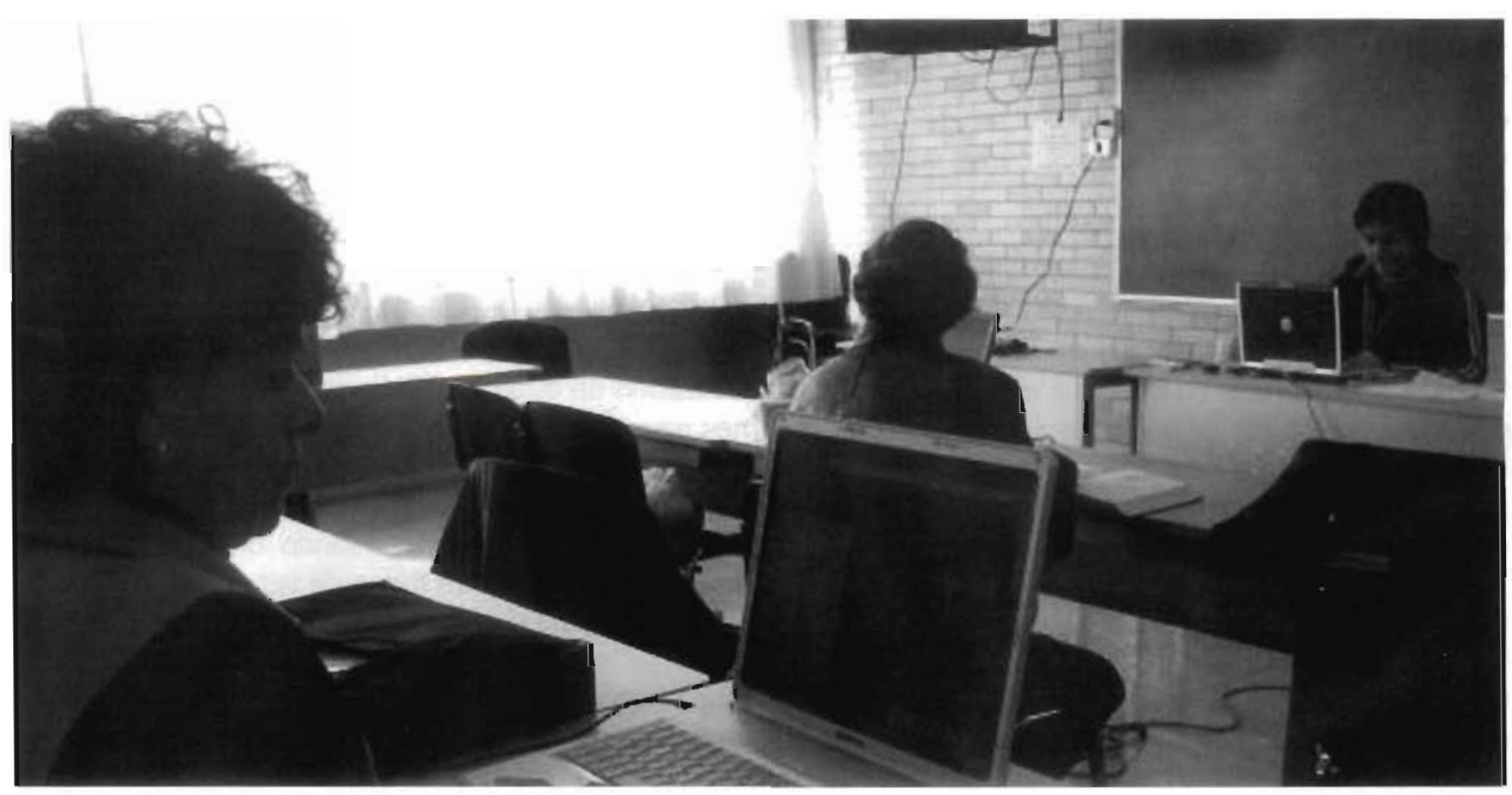

